\title{
windbeutel, a gene required for dorsoventral patteming in D rosophila, encodes a protein that has homologies to vertebrate proteins of the endoplasmic reticulum
}

\author{
Mary Konsolaki and Trudi Schüpbach ${ }^{\mathbf{1}}$ \\ Howard Hughes Medical Institute, Department of Molecular Biology, Princeton University, \\ Princeton, N ew Jersey 08544 USA
}

\begin{abstract}
The formation of the dorsoventral axis of the D rosophila embryo depends on cell-cell interactions that take place in the female ovary and involve the activation of transmembrane receptors by secreted ligands. The gene windbeutel functions in the somatic follicle cells of the ovary and is required for the generation of a signal that will determine the ventral side of the embryo. This signal originates in the follicle cells during oogenesis, but its actions are only manifested after fertilization, when the egg has al ready been laid. We have performed a molecular analysis of $w$ indbeutel. We have found that $w$ indbeutel encodes a putative resident protein of the endoplasmic reticulum, and has homologs in rats and humans. The gene is expressed for a brief period of time in the follicle cells of the ovary, at around the time when the dorsoventral axis of the egg chamber is first established. We propose that Windbeutel is responsible for the folding and/or modification of a specific factor that is secreted from the follicle cells and participates in the activation of the ventralizing signal.
\end{abstract}

[Key Words: Drosophila; pattern formation; oogenesis; endoplasmic reticulum; secretion]

Received September 5, 1997; revised version accepted N ovember 3, 1997.

In Drosophila, the establishment of dorsoventral polarity occurs during oogenesis and requires communication between the germ-line-derived oocyte and the somatically derived follicle cells of the ovary. Initially, the oocyte sends a dorsalizing signal to the follicle cells. This signal is received by the follicle cells via Egfr, the Drosophila homolog of the human epidermal growth factor receptor (Wadsworth et al. 1985; Price et al. 1989; Schejter and Shilo 1989). Egfr, which is expressed in the follicle cells, is believed to be locally activated by Gurken (Grk), a TGF $\alpha$ type molecule, produced by the oocyte (N euman-Silberberg and Schüpbach 1993). Egfr activati on by Grk takes place on the dorsal side of the follicular epithelium and regulates a second process of cell communication, relating dorsoventral information from the follicle cells back to the egg. Ultimately, the signal from the follicle cells leads to the specification of the dorsoventral axis of the embryo.

${ }^{1}$ Corresponding author.

E-MAIL gschupbach@mol biol.princeton.edu; FAX (609) 258-1547.
This second signal ing process from the follicle cells to the embryo requires the action of 11 genes that belong to the dorsal group of maternal-effect genes. Loss-of-function mutations in these genes lead to a dorsalization of the embryo. According to current models, the signal from the follicle cells results in the activation of the Toll receptor only on the ventral side of the egg, and ultimately leads to the formation of the nuclear gradient of the transcription factor Dorsal (for review, see M orisato and Anderson 1995). The activation of the Toll receptor is mediated by the product of the gene spätzle, whose cleaved, active form is thought to be present only on the ventral side of the embryo (M orisato and Anderson 1994; Schneider et al. 1994). Spätzle is itself activated by processing through a proteolytic cascade of serine proteases encoded by the genes gastrulation defective, snake, and easter.

Three of the dorsal group genes, windbeutel (wind), nudel, and pipe, have been shown by genetic mosaic experiments to be required in the somatic follicle cells rather than in the germ line (Schüpbach et al . 1990; Stein 
et al. 1991). These genes are therefore candi dates for encoding proteins that may directly produce the ventral signal in the follicle cells. The gene nudel has been cloned and shown to encode a modular protein with an extracellular matrix domain and a serine protease domain (Hong and Hashimoto 1995). It has been suggested that $\mathrm{N}$ udel is secreted by the follicle cells and may possibly be incorporated in the vitelline membrane, thus specifying the site of generation of the active Spätzle ligand, after fertilization of the oocyte (Hong and Hashimoto 1995). This idea of a localized protease would fit well with the model of proteolytic activation of the ligand for the Toll receptor by sequential processing of a series of serine protease pro-enzymes (for review, see Morisato and Anderson 1995).

The gene Egfr is also required in the somatic follicle cells, and when mutant, causes the generation of ventralized phenotypes in eggs and embryos. Epistasis experiments have shown that the Grk-Egfr pathway negatively regulates the production of the ventral izing signal in the follicle cells, although the exact nature of this interaction remains to be shown (Schüpbach 1987; Roth and Schüpbach 1994). In particular, double mutants between Egfr and wind give rise to dorsalized progeny (Schüpbach et al. 1990). Therefore, it appears that wind acts downstream of Egfr, and that the Grk-Egfr signaling may directly, or indirectly, be responsible for the restriction of Wind activity to the ventral side of the follicular epithelium.

The folliclecel Is form a single-cell epithelial layer that surrounds the nurse cells and the developing oocyte. Their role during oogenesis is multifunctional as they are responsible for the secretion of both structural components of the egg, like the chorion, vitell ine membrane, and yolk proteins (for review, see Spradling 1993), and regulatory molecules like $\mathrm{Nudel}$ (Hong and Hashimoto 1995) or Torso-like (Stevens et al . 1990; Savant-Bhonsale and Montell 1993), that participate in the establishment of embryonic polarity (for review, see Ray and Schüpbach 1996). Thus, the follicle cells form a population of cells highly secretory in nature and are, in addition, the recipients and transmitters of extracellular signals.

To elucidate the mechanism by which the folliclecel Is provide ventral information, we have undertaken the molecular analysis of the gene wind. We show that wind encodes a protein that is a putative resident of the endoplasmic reticulum (ER). It is well documented that the ER plays a role in the translocation, folding, and degradation of membrane bound and secreted proteins (for review, see Rapoport et al. 1996). Our findings lead us to propose that the product of the gene wind is specifically involved in the modification and/or folding of a factor secreted by the follicle cells over the ventral side of the oocyte. This factor is then responsible for the activation of the proteolytic cascade, and possibly for its restriction to the ventral side of the egg. The cascade will rel ease the active Spätzl e ligand that will subsequently bind and activate the Toll receptor. The proposed model is based on the temporal expression pattern of wind as well as on the structural motifs present on the putative Wind protein.

\section{Results}

Phenotypic analysis of the gene wind

The gene wind was identified in a screen for female sterile mutations (Schüpbach and Wieschaus 1989). Females homozygous for wind mutations produce eggs that have normal morphology but give rise to embryos that are dorsalized. At the end of embryogenesis, such embryos secrete a dorsalized cuticle, which is characterized by the absence of ventral structures such as ventral denticle bands, and the expansion of dorsal epidermal structures. The severity of the cuticle phenotype varies depending on the allelic combination, ranging from weakly dorsalized (D2), to strongly dorsalized (D0) (Fig. 1; for a definition of classes of dorsalization, see Anderson et al . 1985). The maternal defect, caused by wind mutations, cannot be rescued zygotically.

We have characterized the existing six EMS induced wind alleles in trans to deficiencies that remove the gene and also in all possible trans-heterozygous combinations (Table 1). Five of these alleles show reduced viability in adults, surviving at a rate ranging from $8 \%-62 \%$ of the expected progeny. Females homozygous for these alleles give rise to strongly dorsalized progeny, $100 \%$ of which
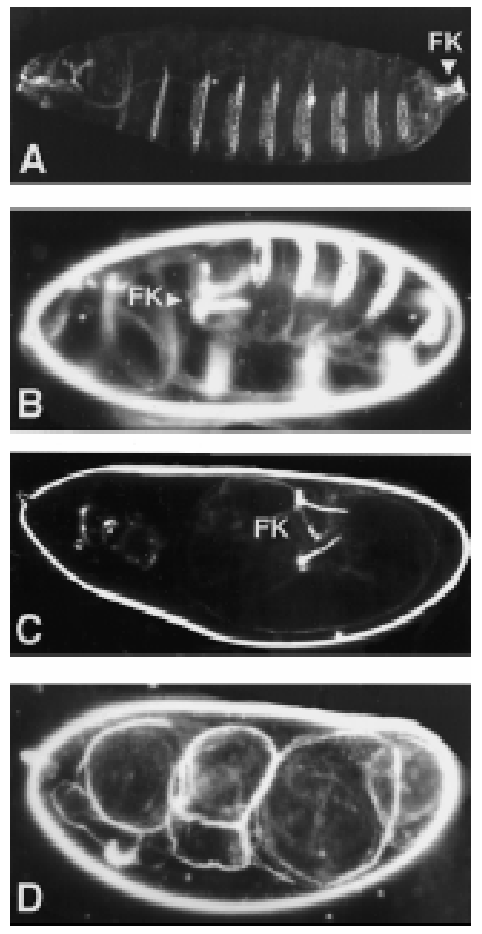

Figure 1. Embryonic cuticles. (A) Wild type embryo with normal head structures, three thoracic segments, eight abdominal segments carrying ventral denticle belts and tel son [Filzkörper (FK)]. (B) Weakly dorsal ized embryo (D2), in which devel opment has arrested at the extended germ-band stage, causing the production of an elongated, u-shaped embryo with Filzkörper (FK) on the dorsal side. (C) M ore dorsal ized embryo (D1), still exhibiting Filzkörper structures. (D) Extremely dorsalized embryo (D0). All embryos are shown inside the vitelline membrane. 
Table 1. Complementation analysis of wind mutations

\begin{tabular}{|c|c|c|c|c|c|}
\hline \multirow[b]{2}{*}{ Alleles } & \multirow{2}{*}{$\begin{array}{l}\text { Percent } \\
\text { viability }\end{array}$} & \multirow{2}{*}{$\begin{array}{l}\text { Percent } \\
\text { hatch }\end{array}$} & \multicolumn{3}{|c|}{$\begin{array}{c}\text { Phenotype of unhatched } \\
\text { progeny }(\%)\end{array}$} \\
\hline & & & D0 & D1 & D2 \\
\hline RP54/M 46 & 8 & 0 & 100 & & \\
\hline RP54/M 88 & 13 & 0 & 100 & & \\
\hline RP54/T 6 & 27 & 0 & 100 & & \\
\hline RP54/E4 & 16 & 0 & 100 & & \\
\hline RP54/AR51 & 32 & 0 & & 60 & 40 \\
\hline M 46/M 88 & 14 & 0 & 100 & & \\
\hline M 46/T6 & 60 & 0 & 100 & & \\
\hline M 46/E4 & 43 & 0 & 100 & & \\
\hline M 46/AR51 & 49 & 0 & 100 & & \\
\hline M 88/T6 & 24 & 0 & 100 & & \\
\hline M 88/E4 & 40 & 0 & 100 & & \\
\hline M 88/AR51 & 62 & $\sim 99$ & N.A. & N.A. & N.A. \\
\hline T6/E4 & 19 & 0 & 100 & & \\
\hline T6/AR51 & 32 & 0 & & 50 & 50 \\
\hline E4/AR51 & 33 & 0 & & & 100 \\
\hline AR51/AR51 & 100 & 0 & & & 100 \\
\hline RP54/DfP34 & 47 & 0 & 100 & & \\
\hline M 46/DfP34 & 31 & 0 & 100 & & \\
\hline M 88/DfP34 & 30 & 0 & 100 & & \\
\hline T6/DfP34 & 47 & 0 & 100 & & \\
\hline E4/DfP34 & 18 & 0 & 100 & & \\
\hline AR51/DfP34 & 58 & 95 & & & 100 \\
\hline
\end{tabular}

(N.A.) N ot applicable. show the mutant phenotype. The sixth allele (wind ${ }^{A R 51}$ ) appears weaker because it shows better viability in trans to the rest of the alleles and to the deficiency, and gives rise to milder dorsalized phenotypes (Table 1 ).

The lethal phase for three of the stronger alleles $\left(\right.$ wind $^{\mathrm{RP} 54}$, wind ${ }^{\mathrm{M} 88}$, and wind $^{\mathrm{T} 6}$ ) was determined to be between the second and third instar larval stages. This suggests that wind is also required zygotically, and functions in at least one other stage of development.

\section{Molecular analysis of the wind locus}

By mei otic mapping, wind was positioned at 2-86 on the second chromosome (Schüpbach and Wieschaus 1989). Complementation tests, using various chromosomal deficiencies, showed that wind is uncovered by the $\mathrm{Df}(2 \mathrm{R}) \mathrm{P} 34$, which spans the region $55 \mathrm{E}-56 \mathrm{C}$ and by the $\mathrm{Df}(2 \mathrm{R}) \mathrm{GC}$, which maps in the proximal $56 \mathrm{C}$ region and uncovers the coracle (cor) locus (Fehon et al. 1994). The results of the deficiency analysis are shown in Figure 2A.

Using the P-element insertion P989 (Karpen and Spradling 1992), we were able to determine that $\mathrm{Df}(2 \mathrm{R}) \mathrm{GC} 8$ extends more distally than Df(2R)P34 (Fig. 2A). In addition, we created wild-type chromosomes by recombination between $\operatorname{cor}^{1}$ and wind ${ }^{\mathrm{RP} 54}$, and with the help of flanking markers, showed that wind maps distal to cor. We tested a total of 60,000 chromosomes and found that
Figure 2. Analysis of the genomic region containing wind. (A) Deficiency mapping of the 55A-56C area and complementation analysis of wind. The P-element insertion P989 is also indicated. (B) Schematic representation of the genomic walk in the 56C area. The total size of the area depicted is $\sim 90 \mathrm{~kb}$. Black horizontal bars be low the solid line represent the fragments that were used as probes in the reverse $\mathrm{N}$ orthern and $\mathrm{N}$ orthern analyses. Beneath the probes, the sizes of transcripts that were detected are indicated. (nt) No transcript. The 6-kb transcript corresponds to the cor transcription unit and the $2.5-\mathrm{kb}$ transcript was present only in RNA from male flies. Boxed are the transcripts that were expressed in ovaries. Also shown are the fragments used in the rescue construct (hatched line), and the area where the distal breakpoint of deficiency Df(2R)P34 maps (thick broken line).

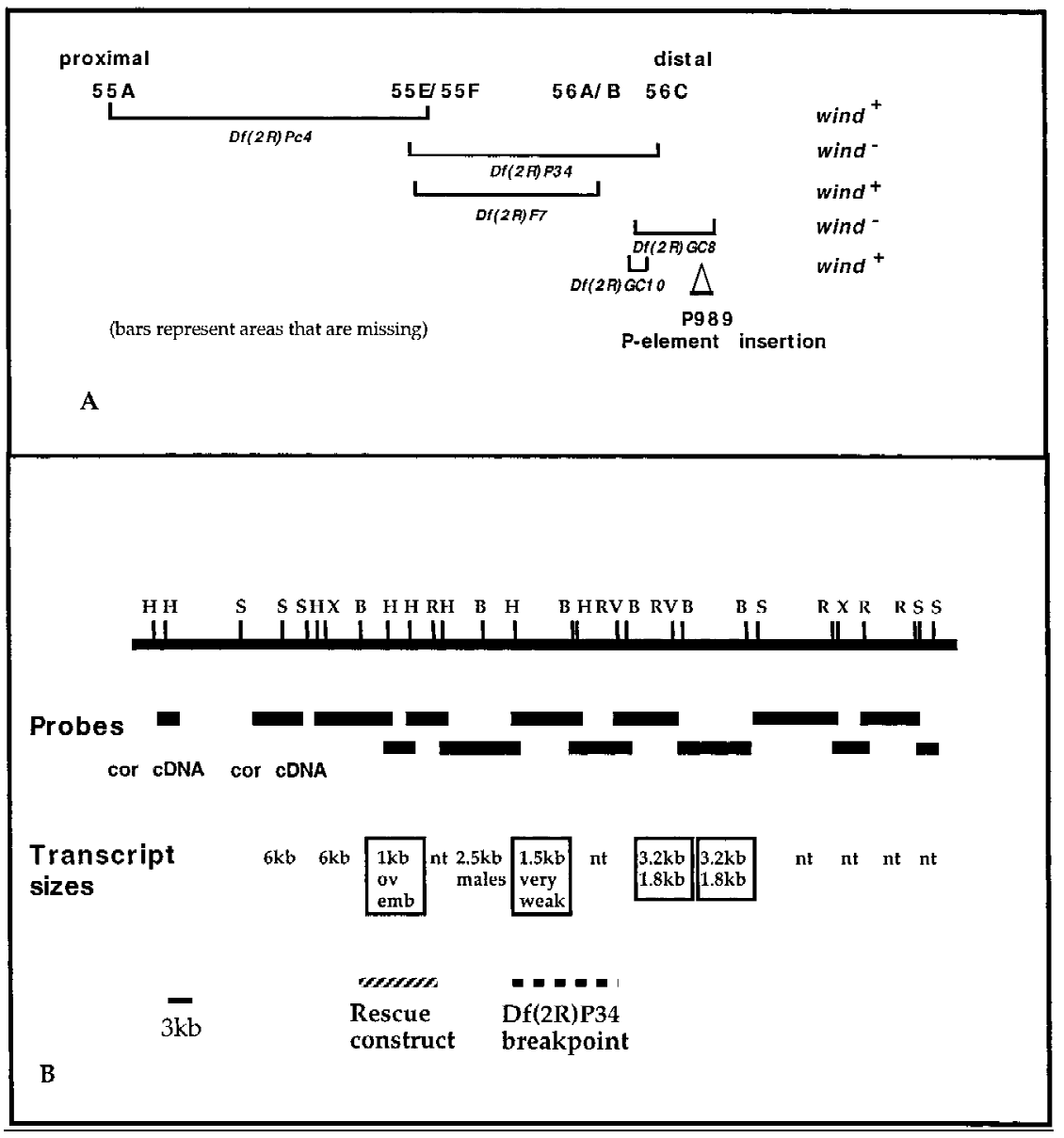


three independent recombination events gave rise to wild-type chromosomes. The distance between the two genes was calculated by the frequency of the single recombinations and was estimated to be $0.01 \mathrm{cM}$ (Ashburner 1989). On the basis of these cal culations, we concluded that the wind gene should lie very close to coracle.

We generated a chromosomal walk on a $\lambda$ phage genomic library, using as probes a cDN A fragment from the cor gene for the proximal end and a genomic fragment adjacent to the P-element insertion P989 for the distal end (see Materials and Methods). Part of the genomic area represented in the chromosomal walk is covered by overlapping fragments from a P1 phage (DM 02733) that we mapped in the relevant area. The chromosomal walk covers a total of $90 \mathrm{~kb}$ of genomic DNA (Fig. 2B). The distal breakpoint of Df(2R)P34 was also mapped by Southern analysis (Fig. 2B).

To identify the transcription units that are present in the candidate genomic area, we performed reverse $\mathrm{N}$ orthern and $\mathrm{N}$ orthern analyses, by use of poly $(\mathrm{A})^{+} \mathrm{RN} A$ from ovaries. Three candidate transcription units were detected, shown boxed in Figure 2B. All of these transcripts were expressed in ovaries, as determined by Northern analysis. In situ RNA hybridization analysis on ovaries, by use of genomic DNA fragments corresponding to these transcripts as probes, was also performed. The 3.2-/ 1.8-kb set of alternatively spliced transcripts was found to hybridize predominantly to RN A in the nurse cells. The 1.5-kb transcript gave no detectable signal on ovaries. Given that the N orthern hybridization signal from this particular transcript was extremely low, it is possible that in situ hybridization was not sensitive enough to detect it. Finally, the 1-kb transcript was expressed mainly in the follicle cells, thus it was selected as the most promising candidate for the wind gene.

Subsequently, a rescue construct was built, containing $\sim 6 \mathrm{~kb}$ of DNA (hatched bar in Fig. 2B), surrounding this 1-kb transcript. After standard P-element-mediated transformation (Spradling 1986), we obtained six independent transformed lines. Three of the lines, which had integrated the P-element in the first or third chromosome, were crossed into a wind mutant background and all three insertions were found to rescue the female sterility conferred by the wind mutation. wind mutant females carrying the transgene were also shown to be $100 \%$ viable, as compared with their siblings without the transgene. Thus, one copy of the the 1-kb transcript fully rescues the mutant phenotype, which shows that this transcript corresponds to the wind gene.

The wind gene is expressed during mid-oogenesis in the follicle cells

Expression of the wind transcript was tested by $\mathrm{N}$ orthern blot analysis. The wind transcript is present in ovaries, early embryos (0-4 hr), and adult males but is almost undetectable in female carcasses (Fig. 3).

Previously, it has been shown by genetic mosaics that

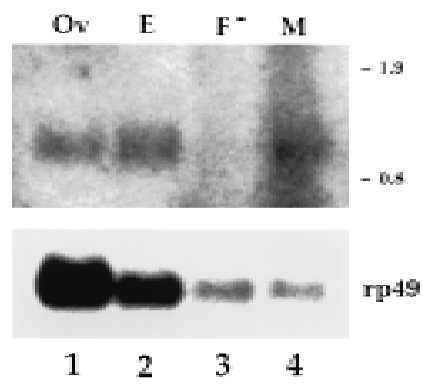

Figure 3. N orthern analysis of RNA from wild-type tissues. The probe used was the genomic fragment that hybridizes to the $1-k b$ transcript shown schematically in Fig. 2B. Poly(A) ${ }^{+}$-selected wild-type RN A was prepaped from ovaries (Ov), 0- to 4-hr embryos (E), females without their ovaries $\left(F^{-}\right)$, and males $(M)$. RN A (15-18 $\mu \mathrm{g})$ was loaded in lanes 1 and 2, $11 \mu \mathrm{g}$ in lane 3, and $7 \mu \mathrm{g}$ in lane 4 . The same blot was al so hybridized to the rp49 probe to provide a loading standard. The numbers at the right correspond to RNA molecular size standards.

the activity of the gene wind is required in a somatic cell type, most likely in the follicle cells of the ovary (Schüpbach et al. 1990; Stein et al. 1991). To examine the expression pattern of wind in oogenesis, we performed RNA in situ hybridization on ovaries. Expression is not detected in the germarium or early stage egg-chambers (Fig. 4A) and is first detectable in the follicle cells of stage 8 egg-chambers. The peak of expression occurs in the follicle cells of stages 9 and early 10, and the transcript di sappears completely before stage 11 (Fig. 4B-D). The germ-line cells (nurse cells and oocyte) do not express any wind RN A, with the possible exception of late stage 10 nurse cells.

Careful examination of the wind expression pattern reveal ed that only the follicle cells that are located over the oocyte express the wind transcript, in contrast to the foll icle cells covering the nurse cells (Fig. 4B). Within the columnar follicle cell epithelium, there does not seem to be any restriction of wind expression relative to the dorsoventral axis. Rather, all the follicle cells over the oocyte are uniformly expressing wind. Interestingly, the transcript appears to be concentrated in the apical cytoplasm of the follicle cells, which in the follicular epithelium is the side facing the oocyte. The functional significance of this localization has not yet been examined but we observed that in situ hybridization by use of two different probes (nudel and cor), which are al so expressed in the follicle cells, did not show a similar pattern (data not shown).

We also performed RNA in situ analysis on ovaries from flies hemizygous for the different wind alleles (data not shown). wind RNA was detected in the four of the hemizygous mutant alleles, three of which give a strong dorsalized phenotype (wind ${ }^{\mathrm{RP} 54}$, wind ${ }^{\mathrm{T} 6}$, and wind $^{\mathrm{E} 4}$ ) and in the weak allele wind ${ }^{\mathrm{AR} 51}$. In two of the alleles (wind $^{\mathrm{M} 88}$ and wind $^{\mathrm{M} 46}$ ), wind RNA expression was reduced to an almost undetectable level. Given the limitations of the in situ hybridization sensitivity, it is not 


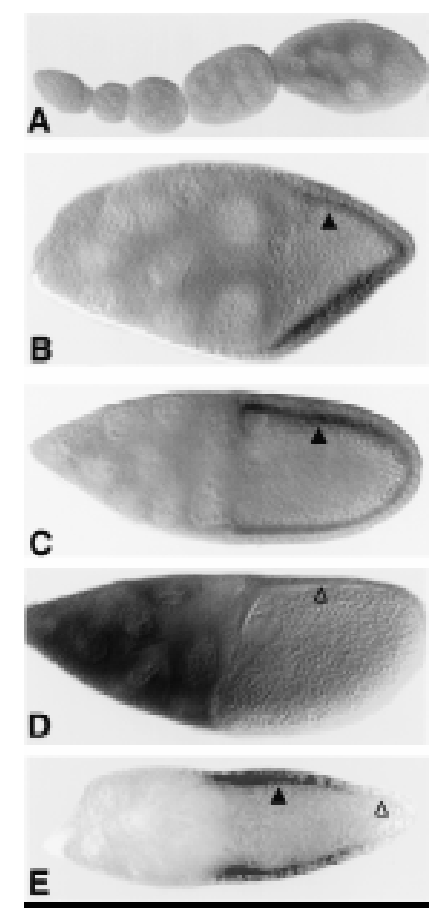

Figure 4. Expression and localization pattern of wind RNA in oogenesis. In situ hybridization on whole-mount ovaries was carried out by use of a wind antisense RN A probe corresponding to the PCR product of the $5^{\prime}$ half of the wind gene. Staining was visualized by an alkaline phosphatase secondary antibody. (AD) Wild-type egg chambers. (E) Egg chamber mutant for the grk gene. (A) Egg chambers at stages 1-8, wind is not expressed. (B) Egg chamber in stage 9, wind RNA accumulates on the apical side of the follicle cells surrounding the oocyte $(\boldsymbol{\Lambda})$, it is not expressed in the follicle cells lying over the nurse cells. (C) Egg chamber at stage 10A, wind RN A still accumulates at the apical end of the follicle cells (A). (D) Egg chamber at late stage 10B, wind RNA is no longer detected in the follicle cells $(\triangle)$. There is some staining seen in the nurse cells at this stage. It should be noted, however, that nurse cells of that stage have been shown to absorb a great variety of different probes (Spradling 1993); thus, it is difficult to assess whether this staining corresponds to specific expression of wind. (E) In a grk mutant egg chamber, wind RNA is still expressed in the follicle cells that overlie the anterior part of the oocyte $(\mathbf{\Lambda})$. It is not expressed in the follicle cells overlying the posterior end of the oocyte $(\triangle)$.

possible to assess whether RNA expression is completely abolished in these mutants.

Expression of the wind transcript in mutants affecting the dorsoventral pattern

To examine whether wind RN A expression is affected by mutations in other genes involved in the establ ishment of dorsoventral polarity, we analyzed its expression in different mutant backgrounds. First we examined ovaries mutant for $\mathrm{fs}(1) \mathrm{K} 10$, a gene that when mutant in females, produces dorsalized eggs and embryos (Wieschaus 1979). fs(1)K10 acts upstream of the dorsoventral Grk-Egfr signaling process (for review, see Ray andSchüpbach 1996). In ovaries mutant for fs(1)K10, the ex- pression of wind RNA is not affected. Similarly, wind RNA expression was unaffected in ovaries mutant for nudel and pipe, the other two genes that act at the same step as wind in the dorsoventral pathway (data not shown).

In ovaries mutant for a strong gurken allele, wind expression was eliminated in all follicle cells around the posterior of the egg chamber (Fig. 4E). This observation can be explained by taking into account the earlier role of the Grk-Egfr signaling process in instructing posterior follicle cells, prior to its role in instructing dorsal follicle cells (Gonzales-Reyes and St Johnson 1995; Roth et al. 1995). The posterior signaling process takes place before stage 8 of oogenesis, and is required for posterior follicle cells to take on the posterior fate, rather than a default anterior fate. In grk mutant egg chambers, the posterior follicle cells behave as anterior follicle cells, and at stages 8 to 10, similar to the anterior follicle cells situated over the nurse cells, they do not express wind (Fig. 4E).

In summary, both the spatial and temporal wind RN A expression that we observed in the follicle cells of the egg chamber is consistent with a specific role of wind in dorsoventral patterning during oogenesis.

The wind gene encodes an endoplasmic reticulum protein

Efforts to isolate a wind cDNA from an ovarian library were not successful, although a large number of clones $(\sim 1,200,000)$ were screened from two different ovarian cDN A libraries (provided by Dr. P. Tolias, Public Heal th Research Institute, N ew Y ork, NY, and Dr B. Suter, M cGill University, M ontreal, Canada). This is likely caused by a low abundance of the particular RNA in the total ovarian RNA population. To overcome this difficulty, we first sequenced the area of the genomic DNA that contains the transcript and made appropriate primers from DNA regions containing open reading frames. These primers were used in two separate PCR reactions (see $M$ aterials and $M$ ethods) that amplified the $5^{\prime}$ and the $3^{\prime}$ ends of the transcript in two overlapping fragments. Both the genomic and the CDN A fragments were found to contain identical sequences (Fig. 5). The genomic sequence contained a small intron of $193 \mathrm{bp}$ located after amino acid 92 of the putative protein. It appears that the combined PCR products contain all of the CDNA sequence because the size of the transcribed area (956 bp) corresponds very cl osely to the size of the transcript that was detected on the $\mathrm{N}$ orthern bl ot $(1 \mathrm{~kb})$. Also, the $3^{\prime}$ end PCR product contained a long poly $(A)$ tail, right after a consensus polyadenylation signal present in the genomic sequence. Sequence anal ysis showed that the wind transcript encodes a putative protein of 257 amino acids with an estimated molecular mass of $29 \mathrm{kD}$.

Database similarity searches revealed that the putative protein encoded by wind is homologous to the rat endoplasmic reticulum protein ERp29 precursor (Demmer et al. 1997). The two proteins share $30 \%$ overall identities and $40 \%$ homology, taking conserved changes 


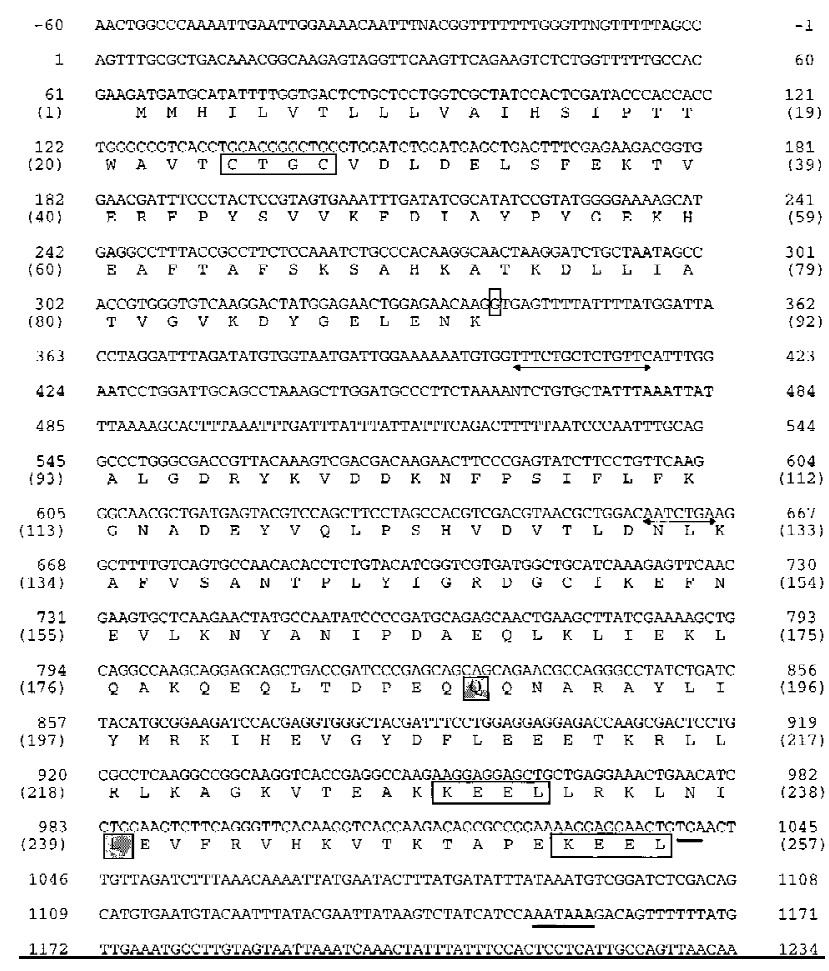

Figure 5. Nucleotide sequence and deduced protein of the wind gene. The sequence of the cDNA was identical to the genomic sequence (outsi de of the intron area). The start of transcription was determined by sequencing of the $5^{\prime}$ of the PCR amplified CDNA. A long poly(A) tail was found in the $3^{\prime}$ end PCR amplified product, after a consensus polyadenylation signal present in the genomic sequence. The flanking numbers correspond to nucleotide and amino acid (in parentheses) positions. The 4-amino-acid ER retention signal (KEEL) and thioredoxin-like motif (CTGC) are boxed. Underlined are the translation stop codon (UGA) and consensus polyadenylation signal (AATAAA ). Underlined with arrowed lines are the 7-bp del etion found in wind ${ }^{\mathrm{M} 88}$ and the 14-bp deletion found in wind ${ }^{\mathrm{AR} 51}$. The amino acid changes in wind ${ }^{\mathrm{M} 46}$ and wind $^{\mathrm{T} 6}$ are boxed and shaded and the base change in the intron of wind ${ }^{\mathrm{AR} 51}$ is boxed.

into account (Fig. 6). The identities cluster in specific fragments located in the amino- and carboxy-terminal ends of the putative proteins. There is al so a human protein (Hughes et al. 1993) that shows homology to the rat ERp29 protein. The three molecules have very similar sizes (fly, 257 amino acids; rat, 260 amino acids; and human, 261 amino acids). The human and the rat proteins are $90 \%$ identical (Fig. 6). The rat protein has been proposed to reside in the ER because it was i solated from the microsomal fraction of the cells (Demmer et al. 1997), and it also contains a variant of the ER retention signal (KEEL, Pelham 1990). Significantly, Wind, like the other two proteins, contains two of those variant ER retention signals, located in comparable positions in the carboxyl terminus of the molecule (Figs. 5 and 6). All three proteins contain putative signal peptides of variable sizes at their amino-terminal end, but no obvious glycosylation sites. The amino-terminal hydrophobic se- quences corresponding to the signal peptides in the three homol ogous mol ecules are not conserved. Outside of the signal peptide area, the putative proteins are mostly hydrophilic.

Secondary structure predictions for the putative Wind protein suggest that the molecule contains two large domains, at the amino and carboxyl termini that form $\alpha$ helical structures. Similar structures are predicted for the rat ERp29 protein (Demmer et al. 1997), leading to suggestions of the ends of the protein being folded as globular domains. This suggestion is supported by comparisons of the rat and human proteins ( $90 \%$ identical), where the nonconserved amino acids are clustered in the signal peptide and carboxy-terminal parts (Demmer et al . 1997).

Other ER resident proteins like protein disulfide isomerase and related molecules, are involved in folding of nascent polypeptides and the isomerization of disulfide bridges (N oiva and Lennarz 1992). This function is mediated by a thioredoxin-like catalytic domain that contains the motif [Cys-X-X-Cys] (N oiva and Lennarz 1992, Chivers et al. 1996). Wind contains a thioredoxin-like motif between amino acids 24-27 [Cys-Thr-Gly-Cys] as well as a single Cys residue in amino acid position 149. The single Cys is also conserved in the rat sequence, ERp29, which displays significant homology (25\% identities over a stretch of 103-122 residues) to two different protein disulfide isomerases, but does not contain any intact thioredoxin-like motif (Demmer et al. 1997). The

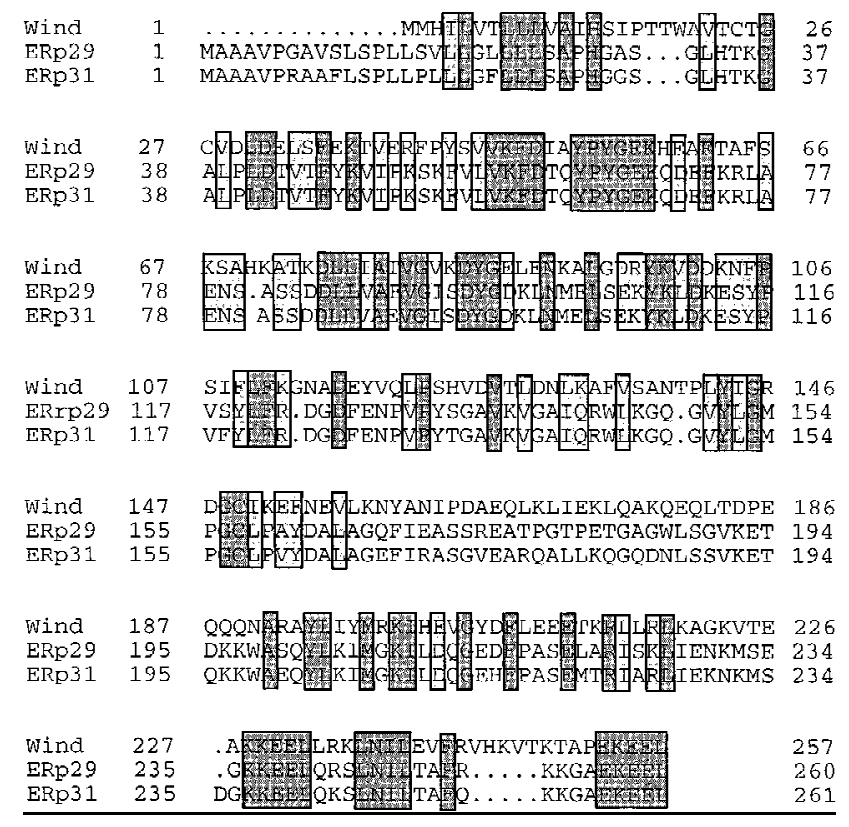

Figure 6. Alignment of the putative amino acid sequences encoded by wind, rat ERp29, and human ERp31. The Drosophila sequence is shorter at the amino-terminal end but outside of this part, the three molecules are al most contiguous. $\mathrm{N}$ ote that the homologous parts cluster in the amino-terminal half and the carboxy-terminal end of the sequences. Identical amino acids in all three sequences are shown by dark shading; conserved changes are shown in lighter shading. 
deduced Wind protein shows 35\% identities to the probable protein disulfide isomerase $\mathrm{P} 5$ precursor from al falfa (Shorrosh and Dixon 1992), over a region of 50 amino acids at its carboxyl terminus. Interestingly, this region is part of the conserved areas between the Drosophila and rat homologs. The rat ERp29 protein is, over the same region, 33\% identical to the alfalfa P5.

\section{Sequence analysis of wind EMS mutants}

To gain additional information regarding the functional domains of the protein, we sequenced some of the wind EMS mutant alleles. Four alleles that were sequenced belong to the group that generates strong dorsalizing phenotypes. They are $100 \%$ penetrant, and confer low viability to mutant flies. The fifth allele sequenced is the weakest hypomorphic allele and it is fully viable. PCRamplified genomic DNA was prepared from flies hemizygous for each of the alleles. Only the transcribed parts of the genomic DNA were sequenced (see M aterials and M ethods).

Sequencing of wind ${ }^{\mathrm{M} 88}$ revealed that the mutant copy of wind contained a 7-bp deletion, between bases 455 and 461 (underlined with arrowed line in Fig. 5). This del etion destroys the reading frame after amino acid 130 and generates a termination codon after another 36 amino acids. Because this deletion occurs in the middle of the coding region, this allele encodes only half of the putative protein, fused to 36 more amino acids of an aberrant sequence. The wind ${ }^{\mathrm{M} 46}$ allel e contains a single base change, resulting in the generation of a stop codon (UAG) after amino acid 187. Thus, this mutant allele also generates a severely truncated molecule. Both the wind $^{\mathrm{M} 88}$ and wind $^{\mathrm{M} 46}$ alleles also displayed a common base substitution, relative to the wild-type sequence. $A$ single base change in nucleotide position 594 results in the conversion of an alanine in the wild-type protein, to a valine in both the mutant proteins. Because both of these alleles were generated on the same parental chromosome, it seems likely that this conserved change represents a polymorphism of the parental allele.

The third allele that was sequenced, wind ${ }^{\top 6}$, also showed a single base change resulting in the conversion of the leucine of amino acid position 239 to a proline (boxed and shaded in Fig. 5). No other change was detected in this allele. Sequencing of the transcribed regi on of the allele wind ${ }^{\mathrm{E} 4}$ did not reveal any changes in the nucleotide sequence. Finally, sequencing of the weakest allele wind ${ }^{\mathrm{AR} 51}$ showed a 14-bp deletion in the intron sequence and a base change of the first nucleotide of the intron (from $G$ to $A$, boxed in Fig.5). A few other base changes were also detected that only affected the third base of codons.

\section{Discussion}

Expression of wind in the follicle cells occurs when the dorsoventral pattern is established

The establishment of polarity in the Drosophila embryo is the result of a network of cellular communication that takes place in the ovary during the process of oogenesis. Both the somatic follicle cells and the germ cells are involved in an intricate exchange of signals, which regulate the polarity of the embryo. Even though the signaling processes are initiated during oogenesis, their activity ultimately leads to the asymetric activation of zygotic genes in the early embryo (Lehmann 1995; Morisato and Anderson 1995; Ray and Schüpbach 1996).

A set of 11 maternal genes participate in a cascade of events that establishes the nuclear gradient of Dorsal protein in the embryo. Loss-of-function mutations in these genes lead to the absence of nuclear Dorsal protein and therefore cause the generation of dorsalized embryos. Together with a twelfth gene, cactus, these genes comprise the dorsal group of genes, and participate in a cascade of events in which dorsoventral information is transmitted from the follicle cells to the embryo. The three genes, wind, nudel, and pipe, are at the top of this cascade, as it has been shown that their action is required in the somatic follicle cells of the ovary (Schüpbach et al. 1990; Stein et al. 1991). The cascade is under the control of the Grk-Egfr pathway, which restricts its action to the ventral side of the egg. This results in the spatially restricted production of the ligand for the Toll receptor, and to the establishment of the Dorsal gradient.

To understand how the Grk-Egfr signaling process is linked to the action of the dorsal group genes, we have undertaken the genetic and molecular characterization of the gene windbeutel. We observed that the gene is expressed during a very brief period in the folliclecells in mid-oogenesis. The timing of wind expression, during stages 9 and early 10, corresponds very well to its expected time of action. The Grk-Egfr signaling process that is required for dorsoventral patterning, is believed to take place during stage 9, after the oocyte nucleus has moved to its dorsal-anterior position during stage 8 (M ontell et al. 1991). Early response genes in the follicle cells, such as kek-1 and rhomboid, begin to show a dorsoventral asymmetry in their expression pattern beginning at stage 9 of oogenesis (Ruohola-Baker et al. 1993; M usacchio and Perrimon 1996). The gene nudel has al so been shown to be expressed during stage 10 of oogenesis (Hong and Hashimoto 1995).

Even though it is not clear at this point whether the Grk-Egfr signaling process regulates the activity of either wind or nudel directly, the fact that they are expressed at this specific time in oogenesis suggests that their activity is required in the follicle cells close to the time when Egfr activation occurs. Given that this is the stage when the vitelline membrane, the innermost layer of the eggshell, is secreted, this time of expression is consistent with models proposing that asymmetrically distributed dorsoventral factors might be deposited in the vitelline membrane (Manseau and Schüpbach 1989; Stein et al. 1991; Hong and Hashimoto 1995).

\section{Regulation of wind expression}

To examine whether wind transcription is regulated by any of the other genes acting in the dorsoventral path- 
way, we analyzed its expression in mutant backgrounds. This anal ysis showed that $\mathrm{fs}(1) \mathrm{K} 10$, which acts upstream of grk in dorsoventral signaling, and is involved in grk RNA localization (N euman-Silberberg and Schüpbach 1993), does not alter the expression of wind. Expression of wind in a strong grk mutant was abolished in the posteriorly localized follicle cells. It has been shown previously (Gonzales-Reyes and St Johnston 1995; Roth et al. 1995) that Grk-Egfr signaling is required at two times during oogenesis. Grk-Egfr signaling is first required to specify the posterior follicle cells. The second signaling process, at around stage 9, specifies the dorsal follicle cell fate. In strong grk mutants, the first signaling process does not take place and consequently, the posterior follicle cells assume the anterior fate. In the wild-type egg chamber, wind transcript is found only in the follicle cells overlaying the oocyte and not in the follicle cells covering the nurse cells. The absence of wind expression in the posterior follicle cells in grk mutations shows that Grk-Egfr signaling is necessary in posterior follicle cells to express wind. This signaling process, however, is not necessary for lateral follicle cells to express wind, showing that different subpopulations of follicle cells require different inputs to express wind.

$M$ utations in the gene nudel had no effect on wind RNA expression. Because the N udel protein seems to be secreted (Hong and Hashimoto 1995) and Wind protein is probably retained in the cell (see below), it is reasonable to suggest that nudel lies downstream of wind. wind expression was also unchanged in pi pe mutants, suggesting that this gene may also act downstream or at the same step in the pathway as wind.

Specific role of the endoplasmic reticulum of the follicle cells in dorsoventral patterning

The follicle cells play multifunctional roles during oogenesis. They are responsible for the secretion of yolk proteins that are taken up by the oocyte, as well as the secretion of the vitelline membrane and chorion proteins, which form the protective eggshell around the egg (for review, see Spradling 1993). They are al so involved in the signaling processes that hel p establish the anteroposterior and dorsoventral axes of both the oocyte and embryo (for review, see St Johnston 1995; Ray and Schüpbach 1996).

A common characteristic of these different functions of the follicle cells is the secretion of proteins that serve either a structural or a regulatory role. The follicle cells form an epithelial monolayer around the oocyte-nurse cell complex. The apical side of the follicular epithelium faces the oocyte and is the site of secretion. Our findings that the wind transcript is localized on the apical side and encodes a putative (ER) resi dent protein suggest that specific parts of the secretory apparatus in the follicle cells are apical ly local ized. Other examples of transcripts that have been found to have a specific subcellular distribution in epithelial cells include the gene crumbs (Tepass et al. 1990) and the gene wingless (Bar et al. 1994) where subcellular local ization of the RN A has also been linked to its function.

The sequence homology of Wind to the rat ERp29 protein indicates that Wind is most likely a component of the lumen of the ER. Proteins that contain the 4-residue retention signal (KDEL) are found in the ER lumen and are called reticuloplasmins (Koch 1990). These proteins are transferred into the ER with the help of their signal peptide and are retained there through an interaction of the retention signal with a specific receptor (Pelham 1990, Scheel and Pelham 1996). The major reticuloplasmins described to date (calreticulin, endoplasmin, protein disulfide isomerase, and BiP; Koch 1990; Lucero 1994) are all calcium-binding proteins. They are proposed to function as molecular chaperons during protein assembly and degradation (N igam et al . 1994) and as calcium buffers (Hubbard 1996). ERp29 was shown to be unresponsive to cellular stresses that induce known heat shock proteins that are related to members of the reticuloplasmin family, and it is not a calcium-binding protein (Demmer et al. 1997). These results suggest that ERp29 and its Drosophila and human homologs form a new group of reticuloplasmins with a conserved, but presently unknown, function.

It is interesting to note that ERp29 protein was isolated from rat enamel cells involved in dentition, and wind is expressed in ovarian follicle cells, both of which are highly secretory cell types. Determining the targets of Wind in the follicle cells should help to elucidate the novel function of these conserved proteins.

\section{Mutations in wind affect coding regions of the protein}

Strong mutant alleles of wind cause varying degrees of semi-lethal ity that is manifested during late larval stages of devel opment. These al leles appear to be strong loss of function, as judged by genetic tests. Because many of the important regulatory genes that act during oogenesis have also equally important functions during the adult development of the fly (Spradl ing 1993), we consi dered it possible that the null phenotype of wind might be lethal. The initial allele of the gene wind was identified in female sterile screens (Schüpbach and Wieschaus 1989). Subsequent screens (using as tester a chromosome carrying the original female sterile wind ${ }^{\mathrm{RP5} 5}$ allele), however, did not recover any lethal alleles of wind. In situ RNA analysis on wind mutant ovaries reveal ed that two of the strongest EMS alleles, wind ${ }^{\mathrm{M} 88}$ and wind ${ }^{\mathrm{M} 46}$, had undetectable levels of wind RNA, leading us to suggest that they might represent null alleles. Because the transheterozygous combination of these two mutants is only partially lethal, it appears that even with extremely low, to undetectable, levels of wind RN A, the flies can survive to adulthood.

Two of the strongest wind mutant alleles, wind ${ }^{\mathrm{M} 88}$ and wind ${ }^{\mathrm{M} 46}$, were found to encode severely truncated proteins. A third wind allele, wind ${ }^{\mathrm{T}}$, contains a single amino acid change of a leucine to a proline, in its carboxyl terminus. This change is significant, because this specific leucine is part of a well-conserved stretch of 
amino acids at the carboxyl terminus of all three homologous proteins (Drosophila, rat, and human). Also, proline is a cyclic amino acid that is al most never found in $\alpha$-helical structures, which is the favorable secondary structure prediction for the carboxyl terminus of Wind and its rat and human homologs. Because wind ${ }^{\top 6}$ shows a better viability over the Df(2R)P34 than the two truncation alleles, it appears that wind ${ }^{\mathrm{T} 6}$ may retain some of the function of the molecule.

The weakest allele, wind ${ }^{A R 51}$, revealed changes only in the intron sequences. It is possible that the singl e base change of the first nucl eotide at the $5^{\prime}$ splice site affects the level of correct splicing of wind. If this defect is not $100 \%$ penetrant, it could account for the weak nature of this al lele.

A possible role for Wind protein in the specific folding of a secreted factor?

The ER mediates the translocation of proteins to the membrane and is responsible for the correct folding and chemical modification of secreted or membrane-bound proteins. Several components of the ER have been isolated and specific steps in the process have been characterized (Rapoport et al. 1996). M ore recently, specific developmental functions have been assigned to components of the ER. It has been shown (McKibben and Cartwright, unpubl.), that an alternatively spliced message of the Dtrpl gene is expressed in the ER in a tissue specific manner, that is, only in the male reproductive system. It has al so been proposed that cal megin, a mouse testis-specific ER protein, is responsible for folding one or more sperm-specific proteins that are involved in adhesion of sperm to the zona pellucida (I kawa et al. 1997). Finally, the ER-associating protein Vera was recently implicated in the localization of Vgl RNA in Xenopus oocytes (Deshler et al. 1997).

On the basis of these examples, we would speculate that Wind protein is involved in the specific folding of one or more molecules secreted by the follicle cells. These molecules would be secreted only on the ventral side of the follicular epithelium and would represent the critical factors for the generation of the ventralizing signal that translates into the active ligand for the Toll receptor. Several mechanisms could be proposed that might contribute to the generation of the signal only on the ventral side of the follicular epithelium. The protein expression and/or activity of Wind might be restricted in the ventral follicle cells, in response to the Grk-Egfr signaling. Alternatively, the expression of another factor might be restricted in the ventral follicle cells by the Grk-Egfr pathway, and this factor might be subsequently modified by Wind. In a wind mutant female, the ventral factors would not be active, leading to the absence of functional Toll ligand and absence of nuclear Dorsal protein, and thus to a dorsal ized embryo.

Candidate molecules for these secreted factors could be the products of the genes nudel and pipe. Our in situ hybridization results are consistent with the possibility that both of these genes act downstream of wind. In ad- dition, $\mathrm{N}$ udel protein has been proposed to be secreted from the follicle cells (Hong and Hashimoto 1995). An interesting observation regarding the $\mathrm{N}$ udel amino acid sequence is the fact that it contains a highly conserved serine protease domain with eight cysteines (Hong and Hashimoto 1995). It is proposed that these cysteines might form four intramolecular disulfide bonds within the catalytic pocket, as in chymotrypsin. The fact that Wind contains a putative thioredoxin-like motif, involved in the isomerization of disulphide bridges in nascent polypeptides, raises the intriguing possibility that Wind might be involved in the folding of N udel. Further studies will shed light into the novel finding of the involvement of the ER in the specification of embryonic dorsoventral axis in Drosophila.

\section{Materials and methods}

Fly stocks

Six wind EM S-induced mutations were used in this study. The mutation wind ${ }^{\text {RP54 }}$ is described in Schüpbach and Wieschaus (1989). The mutations wind ${ }^{\mathrm{M} 88}$ and wind $^{\mathrm{M} 46}$ were induced on a cn bw sp chromosome that was tested against wind ${ }^{\mathrm{RP} 54}$, according to standard methods (Clifford and Schüpbach 1989). The mutations wind ${ }^{\mathrm{T} 6}$ and wind ${ }^{\mathrm{E} 4}$ were generated by $U$. M ayer, $\mathrm{R}$. Lehmann, and C. N üsslein-Volhard (unpubl.) and wind ${ }^{A R 51}$ was generated by S. Roth and C. N üsslein-Volhard (unpubl.).

$\mathrm{Df}(2 \mathrm{R}) \mathrm{Pc} 4$ and Df(2R)P34 are described in Lindsl ey and Zi mm (1992). Df(2R)GC8 and Df(2R)GC 10 are described in Gertler et al. (1995). Df(2R)F7 was induced by $X$-rays, screening for reversion events in a strain carrying the $\mathrm{H}_{2} \mathrm{E}$ P-element insertion (provided by J.A. Lepesant, Institut Jacques M onod, Centre $\mathrm{Na}$ tional de la Recherche Scientifique, Paris, France) and was mapped on squashes from salivary gland polytene chromosomes. The $\operatorname{cor}^{1}$ allele was generated by I. Dawson (Fehon et al . 1994). The P-element insertion P989 [I (2) 01103] is described in Karpen and Spradling (1992). The rescue construct was injected in $y$ w flies and cn bw was used for the restriction fragment length polymorphism (RFLP) analysis of the deficiencies. Oregon $\mathrm{R}$ was used as the wild-type stock.

Other strains used were $\mathrm{fs}(1) \mathrm{K} 10$ (Wieschaus 1979), grk $^{\mathrm{HK}}$ (Schüpbach 1987), ndl ${ }^{111}$ (Hong and Hashimoto 1996), and pip $^{664}$ (Anderson and N üsslein-Volhard 1984). All marker mutations and deficiencies are found in Lindsley and Zimm (1992).

\section{Genetic crosses}

For the complementation analysis, crosses were performed at room temperature $\left(22^{\circ} \mathrm{C}\right)$ and were put at $25^{\circ} \mathrm{C}$ at late larval stages to enhance the $\mathrm{Cy}$ phenotype. For the determination of the lethal phase of wind EMS alleles, 300-500 embryos were collected from wind $/+\times$ wind $/ C y O$ crosses and counted through all stages of development. The recessive markers $c$ and px were used for the isolation of wild-type recombinants between b pr c $\operatorname{cor}^{1}$ px sp, and b pr wind ${ }^{\mathrm{RP} 54}$ bw.

\section{Chromosomal walks}

The $\lambda$ phages were isolated from a $\lambda$ Dashll genomic library (constructed by R. Padgett, Waksman Institute, Rutgers U niversity, Piscataway, NJ), by use of a cor CDNA (provided by R. Fehon, Duke University, Durham, NC) as probes, for the proximal end, and genomic DNA adjacent to P989 insertion that was cloned taking advantage of the PZ vector (Mlodzik and Hiromi 1991), for the distal end. The middle part of this genomic area could 
not be cloned from the $\lambda$ library because of the presence of repetitive DNA and was eventually isolated in small fragments from the $\mathrm{P} 1$ phage $\mathrm{DM} 02733$.

The ends of $\mathrm{P} 1$ phages mapping in the $56 \mathrm{C}$ area were cloned by PCR amplification by use of the T 7 and SP6 promoters ( $N$ urminsky and Hartl 1993). The P1 phage DM 02733 was chosen because it overlapped with parts of the $\lambda$ genomic walk. The entire insert of the DM $02733 \mathrm{P} 1$ phage was cloned into the Bluescript vector (Stratagene) by generating mini-plasmid Iibraries with six different restriction enzymes. The minilibraries were kept on grid plates and were used for the isolation of suitable overlapping fragments. The genomic insert of DM 02733 was $60-\mathrm{kb}$ long.

\section{RNA in situ hybridization on whole mount ovaries}

Ovaries dissected and teased apart were fixed and hybridized as described in Tautz and Pfeifle (1989) and modified by Suter and Steward (1991). Digoxigenin-labeled antisense RNA probes were synthesized by use of the RNA genius kit (Boehringer Mannheim). Ovaries were mounted in Aqua-polymount (Polysciences, Inc.).

\section{Molecular techniques}

Genomic DN A was prepared as described in Delidakis and Kafatos (1987), and RNA was isolated as described in Brown and Kafatos (1988). Southern bl ot, N orthern blot, and reverse N orthern blot analyses were performed on Zetaprobe nylon membranes (Bio-Rad). For the reverse $\mathrm{N}$ orthern bl ot, $\sim 5 \mu \mathrm{g}$ of poly(A) ${ }^{+}$ ovarian RNA was labeled by reverse transcription. All other molecular techniques were done according to Maniatis et al. (1989).

The wind CDNA was isolated in two overlapping fragments by 5' RACE and 3' RACE reactions, according to the manufacturer's instructions (GIBCO BRL). In each reaction, $1 \mu \mathrm{g}$ of poly $(A)^{+}$ovarian RNA was used.

Genomic DNA was isolated from flies hemizygous for the wind EMS alleles over Df(2R)GC 8 and was amplified by use of the Advantage CDN A PCR core kit (Clontech).

\section{Sequence analysis}

Sequencing of wild-type DN A was done by automated sequencing (Applied Biosystems) and by the method of Sanger et al. (1977). Sequencing of the mutant DN A was done exclusively by automated sequencing. Sequences were assembled and anal yzed by use of the AssemblyLign and MacVector DN A analysis programs (IBI sequence analysis software). Database searches were performed by the FASTA program (Lipman and Pearson 1985). The wind GenBank accession number is AF025408.

\section{Acknowledgments}

We thank S. Roth, F. Gertler, R. Fehon, I. Dawson, J.-A. Lepe sant, R. Padgett, P. Tolias, B. Suter, and C. Hashimoto for their generous supply of fly stocks and reagents. We are greatful to $T$. Vogt, P. Tolias, L. Nilson, A. N orvel, and A.-M. Queenan for critical reading and comments on the manuscript, and all the members of the Schüpbach and Wieschaus laboratories for support and hel pful discussions. We al so especially thank F. Farber for technical assistance, and J. Curtis for hel p in pinpointing the Df(2R)P34 breakpoint. This work was supported by Howard Hughes Medical Institute and by U.S. Public Health Services grant PO1 CA 41086. M. Konsolaki was supported by American Cancer Society fellowship and later by a fellowship from the
Office for the Promotion of Mental Retardation and Developmental Disabilities, New Jersey Dept. of Human Services.

The publication costs of this article were defrayed in part by payment of page charges. This article must therefore be hereby marked "advertisement" in accordance with 18 USC section 1734 solely to indicate this fact.

\section{References}

Anderson, K.V. and C. N üsslein-Volhard. 1984. Information for the dorsal-ventral pattern of the Drosophila embryo is stored as maternal mRNA. Nature 311: 223-227.

Anderson, K.D., G. Jürgens, and C. N üsslein-Volhard. 1985. Establishment of dorsal-ventral polarity in the Drosophila embryo: Genetic studies on the role of the Toll gene product. Cell 42: 779-789.

Ashburner, M. 1989. Map distance and recombination frequency. In Drosophila: A laboratory handbook. Cold Spring Harbor Laboratory Press, Cold Spring Harbor, NY.

Bar, I.L., B.S. Ledrean, and H. Krause. 1994. Subcellular localization of wingless in the Drosophila embryo. Mol. Biol. Cell 5: 383a.

Brown, N.H. and F.C. Kafatos. 1988. Functional CDNA libraries from Drosophila embryos. J. Mol. Biol. 203: 424-437.

Chivers, P.T., M.C.A. Laboissiere, and R.T. Raines. 1996. The CXXC motif: Imperatives for the formation of native disulfide bonds in the cell. EMBO J. 15: 2659-2667.

Clifford, R.J. and T. Schüpbach. 1989. Coordinately and differentially mutable activities of torpedo, the Drosophila melanogaster homolog of the vertebrate EGF receptor gene. Genetics 123: 771-787.

Delidakis, C. and F.C. Kafatos. 1987. Amplification of a chorion gene cluster in Drosophila is subject to multiple cis-regulatory el ements and to long-range position effects. J. Mol. Biol. 197: 11-26.

Demmer, J., C.M. Zhou, and M.J. Hubbard. 1997. Molecular cloning of ERp29, a novel and widely expressed resident of the endoplasmic reticulum. FEBS Lett. 402: 145-150.

Deshler, J.O., M .I. Highett, and B.J. Schnapp. 1997. Localization of Xenopus $\mathrm{Vgl} \mathrm{mRN} \mathrm{A} \mathrm{by} \mathrm{Vera} \mathrm{protein} \mathrm{and} \mathrm{the} \mathrm{endoplasmic}$ reticulum. Science 276: 1128-1131.

Fehon, R.G., I.A. Dawson, and S. Artavanis-Tsakonas. 1994. A Drosophila homologue of membrane-skeleton protein 4.1 is associated with septate junctions and is encoded by the coracle gene. Development 120: 545-557.

Gertler, F.B., A.R. Comer, J.L. Juang, S.M. A hern, M.J. Clark, E.C. Liebl, and F.M. Hoffmann. 1995. enabled, a dosage-sensitive suppressor of mutations in the Drosophila Abl tyrosine kinase, encodes an Abl substrate with $\mathrm{SH} 3$ domainbinding proteins. Genes \& Dev. 9: 521-533.

Gonzalez-Reyes, A., H. Elliott, and R.D. St Johnston. 1995. Polarization of both major body axes in Drosophila by gurkentorpedo signalling. Nature 375: 654-658.

Hong, C.C. and C. Hashimoto. 1995. An unusual mosaic protein with a protease domain, encoded by the nudel gene, is involved in defining embryonic dorsoventral polarity in Drosophila. Cell 82: 785-794.

- - - 1996. The maternal nudel protein of Drosophila has two distinct roles important for embryogenesis. Genetics 143: 1653-1661.

Hubbard, M.J. 1996. A bundant cal cium homeostasis machinery in rat dental enamel cells. Upregulation of calcium store proteins during enamel mineralization implicates the endoplasmic reticulum in calcium transcytosis. Eur. J. Biochem. 239: 611-623.

Hughes, G.J., S. Frutiger, N. Paquet, C. Pasquali, J.C. Sanchez, 
J.D. Tissot, A. Bairoch, R.D. Appel, and D.F. Hochstrasser. 1993. Human liver protein map: U pdate 1993. Electrophoresis 14: 1216-1222.

Ikawa, M., I. Wada, K. Kominami, D. Watanabe, K. Toshimori, Y. N ishimune, and M. Okabe. 1997. The putative chaperone calmegin is required for sperm fertility. Nature 387: 607611.

Karpen, G.H. and A.C. Spradling. 1992. Analysis of subtelomeric heterochromatin in a Drosophila minichromosome by single P-element insertional mutagenesis. Genetics 132: 737-753.

Koch, G.L.E. 1990. The endoplasmic reticulum and calcium storage. BioEssays 12: 527-531.

Lehmann, R. 1995. Establishment of embryonic polarity during Drosophila oogenesis. Semin. Dev. Biol. 6: 25-38.

Lindsley, D.L. and G.G. Zimm. 1992. The genome of Drosophila mel anogaster. Academic Press, N ew York, NY.

Lipman, D.J. and W.R. Pearson. 1985. Rapid and sensitive protein similarity searches. Science 227: 1435-1441.

Lucero, H.A., D. Lebeche, and B. Kaminer. 1994. ER cal cistorin/ protein disulfide isomerase (PDI). Sequence determination and expression of a cDN A clone encoding a cal cium storage protein with PDI activity from endoplasmic reticulum of a sea urchin egg. J. Biol. Chem. 269: 23112-23119.

Maniatis, T., E.F. Fritsch, and J. Sambrook. 1989. Molecular cloning: A laboratory manual. 2nd ed. Cold Spring Harbor Laboratory Press, Cold Spring Harbor, NY.

Manseau, L.J. and T. Schüpbach. 1989. cappuccino and spire: Two unique maternal effect loci required for both the anteroposterior and dorsoventral patterns of the Drosophila embryo. Genes \& Dev. 3: 1437-1452.

Mlodzik, M. and Y. Hiromi. 1992. The enhancer trap method in Drosophila: Its application to neurobiology. In Methods in neuroscience (ed. P.M. Cann), vol 9, pp 397-414. Academic press, Orlando, FL.

Montell, D.J., H. Keshishian, and A.C. Spradling. 1991. Laser ablation studies of the role of the Drosophila oocyte nucleus in pattern formation. Science 245: 290-293.

Morisato, D. and K.V. Anderson. 1994. The spätzle gene encodes a component of the extracellular signaling pathway establishing the dorso-ventral pattern of the Drosophila embryo. Cell 76: 677-688.

- - . 1995. Signaling pathways that establish the dorsal-ventral pattern of the Drosophila embryo. Annu. Rev. Genet. 29: 371-399.

Musacchio, M. and D. Perrimon. 1996. The Drosophila kekkon genes: Novel members of both the leucine-rich repeat and immunoglobulin superfamilies expressed in the CNS. Dev. Biol. 178: 63-76.

Neuman-Silberberg, F.S. and T. Schüpbach. 1993. The Drosophila dorsoventral patterning gene gurken produces a dorsally localized RNA and encodes a TGF $\alpha$-like protein. Cell 75: 165-174.

Nigam, S.K., A.L. Goldgerg, S. Ho, M.F. Rohde, K.T. Bush, and M.Y. Sherman. 1994. A set of endoplasmic reticulum proteins possessing properties of molecular chaperons includes $\mathrm{Ca}(2+)$-binding proteins and members of the thioredoxin superfamily. J. Biol. Chem. 269: 1744-1749.

N oiva, R. and W.J. Lennarz. 1992. Protein disulfide isomerase: A multifunctional protein resident in the lumen of the endoplasmic reticulum. J. Biol. Chem. 267: 3553-3556.

N urminsky, D.I. and D.L. Hartl. 1993. Amplification of the ends of DNA fragments cloned in bacteriophage P1. BioTechniques 15: 201-208.

Pelham, H.R.B. 1990. The retention signal for soluble proteins of the endoplasmic reticulum. Trends Biochem. Sci.
15: $483-486$.

Price, J.V., R.J. Clifford, and T. Schüpbach. 1989. The maternal ventralizing locus torpedo is allelic to faint little ball, an embryonic lethal, and encodes the Drosophila EGF receptor homolog. Cell 56: 1085-1092.

Rapoport, T.A., B. Jungnickel, and U. Kutay. 1996. Protein transport across the eucaryotic endoplasmic reticulum and bacterial inner membranes. Annu. Rev. Biochem. 65: 271303.

Ray, R.P. and T. Schüpbach. 1996. Intercellular signaling and the polarization of body axes during Drosophila oogenesis. Genes \& Dev. 10: 1711-1723.

Roth, S. and T. Schüpbach. 1994. The relationship between ovarian and embryonic dorsoventral patterning in Drosophila. Development 120: 2245-2257.

Roth, S., F.S. N euman-Sil berberg, G. Barcel o, and T. Schüpbach. 1995. cornichon and the EGF receptor signalling process are necessary for both anterior-posterior and dorsal-ventral pattern formation in Drosophila. Cell 81: 967-978.

Ruohola-Baker, H., E. Grell, T.-B. Chou, D. Baker, L.Y. Jan, and Y.N. Jan. 1993. Spatially localized rhomboid is required for establishment of dorsal-ventral axis in Drosophila oogenesis. Cell 73: 953-965.

Sanger, F., S. Nicklen, and A.R. Coulson. 1977. DN A sequencing with chain termination inhibitors. Proc. Natl. Acad. Sci. 72: 5463-5467.

Savant-Bhonsale, S. and D.J. Montell. 1993. torso-like encodes the localized determinant of Drosophila terminal pattern formation. Genes \& Dev. 7: 2548-2555.

Schejter, E.D. and B.Z. Shilo. 1989. The Drosophila EGF receptor homolog (DER) gene is allelic to faint little ball, a locus essential for embryonic development. Cell 56: 1093-1104.

Schneider, D.S., Y. Jin, D. M orisato, and K.V. Anderson. 1994. A processed form of the spatzle protein defines dorsal-ventral polarity in the Drosophila embryo. Devel opment 120: 12431250.

Schüpbach, T. 1987. Germ line and soma cooperate during oogenesis to establish the dorsoventral pattern of egg shell and embryo in Drosophila melanogaster. Cell 49: 699-707.

Schüpbach, T. and E. Wieschaus. 1989. Female sterile mutations on the second chromosome of Drosophila melanogaster. I. Maternal effect mutations. Genetics 121: 101-117.

Schüpbach, T., R.J. Clifford, L.J. M anseau, and J.V. Price. 1990. Dorso-ventral signaling processes in Drosophila. In Cell-cell interactions in early development (ed. J. Gerhart), pp. 163174. Wiley-Liss Inc., NY.

Sheel, A.A. and H.R.B. Pelham. 1996. Purification and characterization of the human KDEL receptor. Biochemistry 35: 10203-10209.

Shorrosh, B.S. and R.A. Dixon. 1992. Molecular characterization and expression of an alfalfa protein with sequence similarity to mammalian ERp72, a glucose regulated endoplasmic reticulum protein containing active site sequences of protein disulfide isomerase. Plant J. 2: 51-58.

Spradling, A.C. 1986. P element mediated transformation. In Drosophila: A practical approach (ed. D.B. Roberts), pp. 175197. IRL Press, Oxford, UK.

- - - 1993. Developmental genetics of oogenesis. In The devel opment of Drosophila mel anogaster (ed. M. Bate, A. M artinez-Arias), pp. 1-70. Cold Spring Harbor Laboratory Press, Cold Spring Harbor, NY.

Stein, D., S., Roth, E. Vogel sang, and C. N üssl ein-Volhard. 1991. The polarity of the dorsoventral axis in the Drosophila embryo is defined by an extracellular signal. Cell 65: 725-735.

Stevens, L.M., H.G. Fronhoffer, M. Klinger, and C. N üssleinVolhard. 1990. Localized requirement for torso-like expres- 
sion in follicle cells for development of terminal anlagen of the Drosophila embryo. Nature 346: 660-662.

Steward, R., S.B. Zusman, L.H. Huang, and P. Schedl. 1989. The dorsal protein is distributed in a gradient in early Drosophila embryos. Cell 55: 487-495.

St Johnston, D. 1995. The intracellular local ization of messenger RN As. Cell 81: 161-170.

Suter, B. and R. Steward. 1991. Requirement for phosphorylation and localization of the Bicaudal-D protein in Drosophila oocyte differentiation. Cell 67: 917-926.

Tautz, D. and C. Pfeifle. 1989. A non-radioactive in situ hybridization method for the localization of specific RN As in Drosophila embryos reveals translation control of the segmentation gene hunchback. Chromosoma 92: 81-85.

Tepass, U., C. Theres, and E. Knust. 1990. crumbs encodes an EGF-like protein expressed on apical membranes of Drosophila epithelial cells and required for organization of epithelia. Cell 61: 787-799.

Wadsworth, S.C., W.S. Vincent III, and D. Bilodeau-Wentworth. 1985. A Drosophila genomic sequence with homology to the human epidermal growth factor receptor. Nature 314: 178180.

Wieschaus, E. 1979. fs(1)K10, a female sterile mutation altering the pattern of both the egg coverings and the resultant embryos in Drosophila. In Cell lineage, stem cell and cell differentiation (ed. N. le Douarin), pp. 291-302. Elsevier/ North-Holland Biomedical Press, N ew York, NY.

Wieschaus, E. and C. N üsslein-Volhard. 1986. Looking at embryos. In Drosophila: A practical approach (ed. D. Roberts), pp. 199-227. IRL Press, Oxford, UK. 


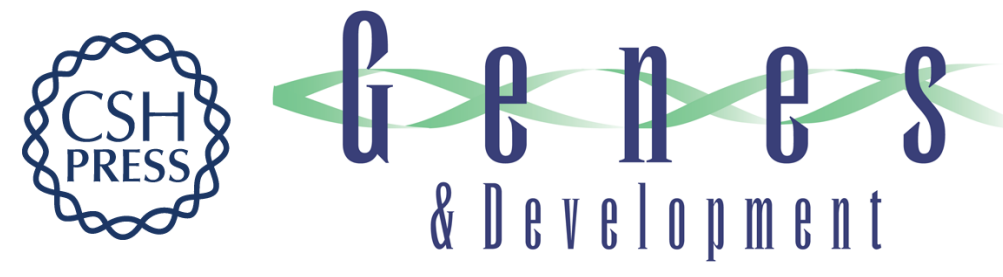

\section{windbeutel, a gene required for dorsoventral patterning in Drosophila, encodes a protein that has homologies to vertebrate proteins of the endoplasmic reticulum}

Mary Konsolaki and Trudi Schüpbach

Genes Dev. 1998, 12:

Access the most recent version at doi:10.1101/gad.12.1.120

References This article cites 51 articles, 16 of which can be accessed free at: http://genesdev.cshlp.org/content/12/1/120.full.htmI\#ref-list-1

License

Email Alerting Receive free email alerts when new articles cite this article - sign up in the box at the top Service right corner of the article or click here.

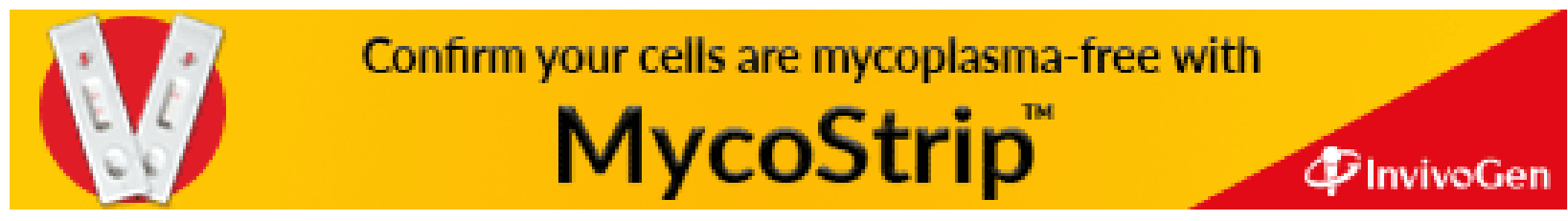

